\title{
INEQUALITIES FOR THE HARTLEY-FOURIER COSINE POLYCONVOLUTION
}

\author{
Phi Thi VAn Anh and Nguyen Xuan ThaO
}

Abstract. In this paper we present some new inequalities of Young's type and Saitoh's type for the Hartley-Fourier cosine polyconvolution. The inequalities of these types hold true for the Fourier convolution, but not for any convolutions or polyconvolutions, which are very few in the literature. We then use these inequalities to estimate the solution of certain integral equations and differential equations.

Mathematics subject classification (2010): 35A23, 42A38, 42B10, 44A35.

Keywords and phrases: Convolution, polyconvolution, Fourier transform, Fourier cosine transform, Hartley transform, integral equation, integral inequalities.

\section{REFERENCES}

[1] R. A. Adams and J. J. F. Fourier, Sobolev Spaces, 2nd ed, Academic Press, 300 p, 2003.

[2] R. N. BRACEWEll, The Hartley transform, Inc, 200 Madison Avenue, New York, 1986.

[3] L. E. Britvina, Polyconvolutions for the Hankel transform and differential operators, Doklady Mathematics, 65, 1 (2002), 32-34.

[4] L. E. BRitvina, On polyconvolutions generated by Hankel transforms, Mathematical Notes, 76, 1 (2004), 18-24.

[5] F. D. GaKhov and Yu. I. Cers KiI, Equations of Convolution Type, Nauka, Moscow, 1978.

[6] V. A. KAKICHEV, Polyconvolution, Taganrog, TPTU, 54p (in Russian), 1997.

[7] N. M. KHOA AND T. TUAn, On the polyconvolution with weight function for Fourier cosine, Fourier and Fourier sine transforms, Advances in Nonlinear Variational Inequalities, 14, 1 (2011), 17-30.

[8] V. V. Napalkov, Convolution Equations in Multidimensional Spaces, Nauka, Moscow, 1982.

[9] R. E. A. C. PAley And N. WIEnER, Fourier Transforms in the Complex Domain, AMS, New York, 1934.

[10] I. N. Sneddon, The Use of Integral Transforms, Mc Gray-Hill. New York, 1972.

[11] S. Saitoh, V. K. TUAn AND M. YAmamoto, Reverse weighted $L_{p}$-norm inequalities in convolutions and stability in inverse problems, J. of Ineq. in Pure \& App. Math., 1, 1 (2000), 1-7.

[12] S. Saitoh, V. K. TuAn AND M. YAмамото, Reverse convolution inequalities and applications to inverse heat source problems, J. of Ineq. in Pure \& App. Math., 3, 5 (2002), 1-11.

[13] S. Saitoh, V. K. TuAn And M. Yamamoto, Convolution inequalities and applications, J. of Ineq. in Pure \& App. Math., 4, 3 (2003), 1-8.

[14] I. N. SnedDON, Fourier Transforms, McGray - Hill, New York, 1951.

[15] E. C. Titchmarch, Introduction to the Theory of Fourier integrals, Third Edition, Chelsea publishing Co., NewYork, 1986.

[16] N. X. Thao And N. A. Virchenko, On the Polyconvolution for the Fourier Cosine, Fourier Sine, and the Kontorovich-Lebedev Integral Transforms, Ukrai. Math. Jour., 62, 10 (2010), 1388-1399.

[17] N. X. Thao, N. M. KhoA AND P. T. VAN AnH, On the polyconvolution for Hartley, Fourier cosine and Fourier sine transforms, Integral Transforms and Special Functions, 24, 7 (2013), 517-531.

[18] N. X. Thao, N. M. KhoA And P. T. VAN Anh, Polyconvolution and the Toeplitz plus Hankel integral equation, Electronic Journal of Differential Equations, 2014, 110 (2014), 1-14.

[19] N. X. Thao, V. K. Tuan And H. T. VAn AnH, On the Toeplitz plus Hankel integral equation II, Integral Transforms and Special Functions, 25, 1 (2014), 75-84. 